\title{
New methods of decision making under uncertainty
}

\author{
Sándor Molnár ${ }^{1}$, Ferenc Szidarovszky² \\ ${ }^{1}$ Institute of Mathematics and Informatics, Szent István University, Páter K. u.1., H-2100, Gödöllö, Hungary \\ ${ }^{2}$ Department of Applied Mathematics, University of Pécs, Ifjúság u. 6, H-7624, Pécs, Hungary
}

\section{Email address:}

Molnar.Sandor@gek.szie.hu (S. Molnár),szidarka@gmail.com (F. Szidarovszky)

\section{To cite this article:}

Sándor Molnár, Ferenc Szidarovszky. New Methods of Decision Making Under Uncertainty. International Journal of Economic Behavior and Organization. Special Issue: Recent Developments of Economic Theory and Its Applications. Vol. 3, No. 2-1, 2015, pp. 5-9. doi: $10.11648 /$ j.ijebo.s.2015030201.12

\begin{abstract}
The classical formula of certainty equivalent is reconsidered. Based on a modified proof of the original formula several alternative methods are derived with different orders of magnitude of their errors. This new method is then compared with the classical formula in a computer study showing the advantage of the new approach. Practical applications are also outlined to illustrate the methodology.
\end{abstract}

Keywords: Uncertainity, Certainty Equivalent, Economic Application

\section{Introduction}

Decision making under uncertainty is one of the most important research areas of operations research and it provides important applications in all fields of quantitative sciences. A large class of models includes random variables, so the decisions are made without knowing their actual values, but all possible values and their probabilistic nature are taken into account. There are several alternative techniques to find optimal decisions. Stochastic programming (Kall and Wallace, 1994; Prekopa, 1995) is the most common approach. Multiobjective optimization (Szidarovszky et al., 1986; Molnár and Szidarovszky, 2011) is an alternative method in which optimizing the expected objective function value and minimizing its variance are considered as the objectives. Reduction of variance can also be achieved with various data analytical techniques (Kovács et al., 2014).

Bayesian decision making (DeGroot, 1970) is based on the repeated updating of the probability distribution when new sample elements are available. Uncertainties can also be modeled by fuzzy sets (Bellman and Zadeh, 1970), when the uncertain parameters and quantities are fuzzified and the level of uncertainty of each quantity is represented by an appropriate membership function.

In the economic literature certainty equivalents are defined and they replace the uncertain objectives and constraints. This approach can be considered as the application of the weighting method when the expectations and variances of the stochastic variables are the objective functions (Sargent, 1979). Another possible application concerns extractability of natural resources (Csábrági et al., 2011) and hydrological research concerning groundwater data (Hatvani et al., 2014). A possible direction can be in the application of emission allowance prices over various non-market intervention schemes (Molnár, 2014)

In this paper we will reconsider the classical formula of the certainty equivalent by analyzing the most common proof of this formula. We will show that by the slight modification of this well-known proof more accurate representations can be obtained, which will be compared with the classical formula. Three applications will illustrate the methodology.

\section{Mathematical Models}

Let $\tilde{x}$ be a random variable and $u$ a utility function. Let $\bar{x}=E(\tilde{x})$ and $\sigma^{2}=\operatorname{Var}(\tilde{x})$. If $x$ is any realized value of $\tilde{x}$, then $u(x)$ shows the value of goodness of outcome $x$ for the decision maker. In the economic literature the certainty equivalent of the random outcome is computed as

$$
x^{*}=\bar{x}-\alpha \sigma^{2}
$$

where $\alpha$ is a constant showing the risk acceptance of the decision maker. Since the certainty equivalent is the fixed outcome that the decision maker would accept instead of the gamble, it has to be the solution of equation 


$$
u\left(x^{*}\right)=E(u(\tilde{x}))=\int_{-\infty}^{+\infty} u(x) f(x) d x
$$

where $f$ is the probability density function of $\tilde{x}$. If $u$ is strictly monotonic, then

$$
x^{*}=u^{-1}\left(\int_{-\infty}^{+\infty} u(x) f(x) d x\right)
$$

It is very difficult to use this formula in practical cases, since the density function is not known in most cases, and even if it is known, the presence of the integral makes the use of this formula very difficult. Numerical integration or simulation can be used to find the value of $x^{*}$. In the economic literature formula (1) is shown to be a fairly good approximation of (3), and even in certain special cases they are equivalent to each other. The usual derivation of the approximation (1) is based on the Taylor polynomial approximation

$$
u(x)=u(\bar{x})+u^{\prime}(\bar{x})(x-\bar{x})+\frac{u^{\prime \prime}(\bar{x})}{2}(x-\bar{x})^{2}+R_{3}(x)
$$

where $R_{3}(x)$ is a third order error term. Similarly,

$$
u\left(x^{*}\right)=u(\bar{x})+u^{\prime}(\bar{x})\left(x^{*}-\bar{x}\right)+R_{2}\left(x^{*}\right)
$$

where $R_{2}\left(x^{*}\right)$ is a second order error term. Notice that the certainty equivalent should satisfy equation (2), so by omitting the error terms, we have

$$
u(\bar{x})+u^{\prime}(\bar{x})\left(x^{*}-\bar{x}\right)=u(\bar{x})+u^{\prime}(\bar{x}) E(\tilde{x}-\bar{x})+\frac{u^{\prime \prime}(\bar{x})}{2} \sigma^{2}
$$

therefore after simplification and by assuming that $u^{\prime}(\bar{x}) \neq 0$,

$$
x^{*}=\bar{x}+\frac{u^{\prime \prime}(\bar{x})}{2 u^{\prime}(\bar{x})} \sigma^{2}
$$

If we introduce the notation $\alpha=-\frac{u^{\prime \prime}(\bar{x})}{2 u^{\prime}(\bar{x})}$, then this relation reduces to the well known rule (1).

There is however a problem with this derivation. In (5) the neglected error term is quadratic, which is the order of the last kept term of (4) so the use of this last term is very problematic. In order to avoid this problem we can use quadratic polynomial approximations in both cases. Then (5) is modified as

$$
u\left(x^{*}\right)=u(\bar{x})+u^{\prime}(\bar{x})\left(x^{*}-\bar{x}\right)+\frac{u^{\prime \prime}(\bar{x})}{2}\left(x^{*}-\bar{x}\right)^{2}+R_{3}\left(x^{*}\right)
$$

where the error term $R_{3}\left(x^{*}\right)$ is also of third order. Now equation (6) becomes

$$
u(\bar{x})+u^{\prime}(\bar{x})\left(x^{*}-\bar{x}\right)+\frac{u^{\prime \prime}(\bar{x})}{2}\left(x^{*}-\bar{x}\right)^{2}=u(\bar{x})+u^{\prime}(\bar{x}) E(\tilde{x}-\bar{x})+\frac{u^{\prime \prime}(\bar{x})}{2} \sigma^{2}
$$

which provides a quadratic equation for $\Delta=x^{*}-\bar{x}$,

$$
\frac{u^{\prime \prime}(\bar{x})}{2} \Delta^{2}+u^{\prime}(\bar{x}) \Delta-\frac{u^{\prime \prime}(\bar{x})}{2} \sigma^{2}=0
$$

By assuming that $u^{\prime}(\bar{x}) \neq 0$, it can be rewritten as

$$
\alpha \Delta^{2}-\Delta-\alpha \sigma^{2}=0
$$

If $\alpha=0$, then $\Delta=0$, so $x^{*}=\bar{x}$. Otherwise

$$
\Delta=\frac{1 \pm \sqrt{1+4 \alpha^{2} \sigma^{2}}}{2 \alpha}
$$

If $\sigma^{2}=0$, then there is no uncertainty, so $x^{*}=\bar{x}$ and $\Delta=0$. Therefore the solution with negative sign is taken:

$$
x^{*}-\bar{x}=\Delta=\frac{1-\sqrt{1+4 \alpha^{2} \sigma^{2}}}{2 \alpha}
$$

that is, the certainty equivalent has the form

$$
x^{*}=\bar{x}+\frac{1-\sqrt{1+4 \alpha^{2} \sigma^{2}}}{2 \alpha}
$$

Formula (9) can be generalized in the following way. Let $M_{k}=E\left((\tilde{x}-\bar{x})^{k}\right)$ be the $k^{t h}$ central moment of $\tilde{x}$. By using $m^{\text {th }}$ order Taylor polynomial approximations, equations (4) and (7) are modified as

$$
u(x)=u(\bar{x})+u^{\prime}(\bar{x})(x-\bar{x})+\sum_{i=2}^{m} \frac{u^{(i)}(\bar{x})}{i !}(x-\bar{x})^{i}+R_{m+1}(x)
$$

and

$$
u\left(x^{*}\right)=u(\bar{x})+u^{\prime}(\bar{x})\left(x^{*}-\bar{x}\right)+\sum_{l=2}^{m} \frac{u^{(l)}(\bar{x})}{l !}\left(x^{*}-\bar{x}\right)^{l}+R_{m+1}\left(x^{*}\right)
$$

By expectation and omitting the error terms

$$
E(u(\tilde{x}))=u(\bar{x})+\sum_{i=2}^{m} \frac{u^{(i)}(\bar{x})}{i !} M_{i}=u(\bar{x})+\sum_{l=1}^{m} \frac{u^{(l)}(\bar{x})}{l !}\left(x^{*}-\bar{x}\right)^{l}=u\left(x^{*}\right)
$$

which gives an $m^{\text {th }}$ degree polynomial equation for $\Delta$ :

$$
\sum_{l=1}^{m} \frac{u^{(l)}(\bar{x})}{l !} \Delta^{l}-\sum_{i=2}^{m} \frac{u^{(i)}(\bar{x})}{i !} M_{i}=0
$$

Notice that in the case of $m=2$ this equation reduces to (8), since $M_{2}=\sigma^{2}$. If the constant term is zero, then (12) can be factored as

$$
\Delta\left(\sum_{l=2}^{m} \frac{u^{(l)}(\bar{x})}{l !} \Delta^{l-1}+u^{\prime}(\bar{x})\right)=0
$$


and since $u^{\prime}(\bar{x}) \neq 0, \Delta=0$ is a single root.

If we consider the root loci of equation

$$
\sum_{l=1}^{m} \frac{u^{(l)}(\bar{x})}{l !} \Delta^{l}-K=0
$$

then only one locus passes through the origin, and the value of this locus at

$$
K=\sum_{i=2}^{m} \frac{u^{(i)}(\bar{x})}{i !} M_{i}
$$

gives the value of $\Delta=x^{*}-\bar{x}$, from which the certainty equivalent can be determined as $x^{*}=\bar{x}+\Delta$.

\section{Comparisons}

A simulation study was performed in comparing equation (1) and equation (9). Four density functions were selected in interval $[-1,1], f_{1}(x)=\frac{1}{2} \quad, \quad f_{2}(x)=\frac{1}{2}(x+1)$, $f_{3}(x)=\frac{1}{2}(1-x)$ and $f_{4}(x)=\frac{3}{4}\left(1-x^{2}\right)$, and four utility functions were chosen,

$$
\begin{gathered}
u_{1}(x)=\frac{1}{4}(x+1)^{2}, u_{2}(x)=1-\frac{1}{4}(x-1)^{2} . \\
u_{3}=\frac{1}{2}\left(1+x^{3}\right) \text { and } u_{4}(x)=\frac{1}{2}+\frac{2}{\pi} \tan ^{-1}(x) .
\end{gathered}
$$

Table 1 shows the simulation results, where $x^{*}$ is the true certainty equivalent, $x_{1}^{*}$ is approximation by formula (1) and $x_{2}^{*}$ is approximation by formula (9). If $u(\bar{x})=0$, then neither approximation can be computed, which is indicated by the sign $N / A$ in the table. Notice that $f_{1}$ is constant, $f_{2}$ increases, $f_{3}$ decreases, and $f_{4}$ is mound-shaped, furthermore $u_{1}$ is convex, $u_{2}$ is concave, $u_{3}$ is concave for $x<0$ and convex for $x>0$, and $u_{4}$ is convex for

\begin{tabular}{|c|c|c|c|c|c|c|}
\hline$f(x)$ & $u(x)$ & $x^{*}$ & $x_{1}^{*}$ & $x^{*}-x_{1}^{*}$ & $x_{2}^{*}$ & $x^{*}-x_{2}^{*}$ \\
\hline \multirow{4}{*}{$f_{1}=\frac{1}{2}$} & $u_{1}(x)=\frac{1}{4}(x+1)^{2}$ & 0.1547 & 0.1667 & -0.0120 & 0.1547 & 0 \\
\hline & $u_{2}(x)=1-\frac{1}{4}(x-1)^{2}$ & -0.1547 & -0.1667 & 0.0120 & -0.1547 & 0 \\
\hline & $u_{3}(x)=\frac{1}{2}\left(1+x^{3}\right)$ & 0 & N/A & N/A & N/A & N/A \\
\hline & $u_{4}(x)=\frac{1}{2}+\frac{2}{\pi} \arctan (x)$ & 0 & 0 & 0 & 0 & 0 \\
\hline \multirow{4}{*}{$f_{2}=\frac{1}{2}(x+1)$} & $u_{1}(x)=\frac{1}{4}(x+1)^{2}$ & 0.4142 & 0.4146 & -0.0004 & 0.4142 & 0 \\
\hline & $u_{2}(x)=1-\frac{1}{4}(x-1)^{2}$ & 0.1835 & 0.1667 & 0.0168 & 0.1835 & 0 \\
\hline & $u_{3}(x)=\frac{1}{2}\left(1+x^{3}\right)$ & 0.5848 & 1.000 & -0.4152 & 0.6667 & -0.0819 \\
\hline & $u_{4}(x)=\frac{1}{2}+\frac{2}{\pi} \arctan (x)$ & 0.2943 & 0.2667 & 0.0267 & 0.2679 & 0.0255 \\
\hline \multirow{4}{*}{$f_{3}=\frac{1}{2}(1-x)$} & $u_{1}(x)=\frac{1}{4}(x+1)^{2}$ & -0.1835 & -0.1667 & -0.0168 & -0.1835 & 0 \\
\hline & $u_{2}(x)=1-\frac{1}{4}(x-1)^{2}$ & -0.4142 & -0.4167 & 0.0025 & -0.4142 & 0 \\
\hline & $u_{3}(x)=\frac{1}{2}\left(1+x^{3}\right)$ & -0.5848 & -1.000 & 0.4152 & -0.6667 & 0.0819 \\
\hline & $u_{4}(x)=\frac{1}{2}+\frac{2}{\pi} \arctan (x)$ & -0.2943 & -0.2667 & -0.0267 & -0.2679 & -0.0255 \\
\hline \multirow{4}{*}{$f_{4}=\frac{3}{4}\left(1-x^{2}\right)$} & $u_{1}(x)=\frac{1}{4}(x+1)^{2}$ & 0.0954 & 0.1000 & -0.9046 & 0.0954 & 0 \\
\hline & $u_{2}(x)=1-\frac{1}{4}(x-1)^{2}$ & -0.0954 & 0.1000 & 0.0046 & -0.0954 & 0 \\
\hline & $u_{3}(x)=\frac{1}{2}\left(1+x^{3}\right)$ & 0 & N/A & N/A & N/A & N/A \\
\hline & $u_{4}(x)=\frac{1}{2}+\frac{2}{\pi} \arctan (x)$ & 0 & 0 & 0 & 0 & 0 \\
\hline
\end{tabular}
$x<0$ and concave for $x>0$. So a large variety of function shapes was considered.

Table 1. Simulation Results.

Among the 16 combined cases 10 times the corrected formula (9) gave the exact value computed by using equations (2), and there was no case when (9) was worse than (1).

\section{Applications}

A. Assume that there are two investment opportunities with uncertain profits. However the investing agent has 
estimates of the expected values and variances of the profits when they are considered random variables: $\mu_{1}, \mu_{2}$ and $\sigma_{1}^{2}, \sigma_{2}^{2}$. Let $u(x)$ denote the utility value if the actual profit is $x$, and define $\alpha_{i}=-u^{\prime \prime}\left(\mu_{i}\right) /\left(2 u^{\prime}\left(\mu_{i}\right)\right)$ for $i=1,2$. The certainty equivalents of the two random profits are

$$
x_{i}^{*}=\mu_{i}+\frac{1-\sqrt{1+4 \alpha_{i}^{2} \sigma_{i}^{2}}}{2 \alpha_{i}}
$$

and the agent will select the investment option with the higher $x_{i}^{*}$ value. In the special case of $\mu_{1}=\mu_{2}$, necessarily $\alpha_{1}=\alpha_{2}$, so investment with smaller variance is chosen.

B. Assume an agent has an investment budget B, which is divided between two types of investment with unit profits $\Pi_{1}$ and $\Pi_{2}$. However $\Pi_{1}$ and $\Pi_{2}$ are uncertain with known expectations $\mu_{1}, \mu_{2}$ with $\mu_{1} \geq \mu_{2}$ and known variances $\sigma_{1}^{2}$ and $\sigma_{2}^{2}$. If the agent invests $x$ and $B-x$ in the two investment types, then its overall profit is

$$
P=\Pi_{1} x+\Pi_{2}(B-x)=\left(\Pi_{1}-\Pi_{2}\right) x+\Pi_{2} B
$$

Clearly

$$
\bar{P}=E(P)=\left(\mu_{1}-\mu_{2}\right) x+\mu_{2} B
$$

and

$$
\operatorname{Var}(P)=\sigma_{1}^{2} x^{2}+\sigma_{2}^{2}(B-x)^{2}
$$

By assume that the utility function is $u(P)=P^{2}$, then

$$
\alpha=-\frac{1}{2\left(\left(\mu_{1}-\mu_{2}\right) x+\mu_{2} B\right)}
$$

and from (9) we have

$$
\begin{gathered}
x^{*}=\left(\mu_{1}-\mu_{2}\right) x+\mu_{2} B-\frac{1-\sqrt{1+\frac{\sigma_{1}^{2} x^{2}+\sigma_{2}^{2}(B-x)^{2}}{\left(\left(\mu_{1}-\mu_{2}\right) x+\mu_{2} B\right)^{2}}}}{\frac{1}{\left(\mu_{1}-\mu_{2}\right) x+\mu_{2} B}} \\
=\sqrt{\left(\left(\mu_{1}-\mu_{2}\right) x+\mu_{2} B\right)^{2}+\sigma_{1}^{2} x^{2}+\sigma_{2}^{2}(B-x)^{2}}
\end{gathered}
$$

There is a convex parabola under the square root, so has its maximum either at $x=0$ or at $x=B$. At $x=0$,

$$
x^{*}=B \sqrt{\mu_{2}^{2}+\sigma_{2}^{2}}
$$

and at $x=B$,

$$
x^{*}=B \sqrt{\mu_{1}^{2}+\sigma_{1}^{2}}
$$

Therefore $x=0$, is chosen, that is the entire budget is invested in the second investment type if $\mu_{2}^{2}+\sigma_{2}^{2}>\mu_{1}^{2}+\sigma_{1}^{2}$, that is, when the second moment of $\Pi_{2}$ is larger than that of $\Pi_{1}$. Similarly $x=B$ is chosen if the second moment of $\Pi_{1}$ is larger than that of $\Pi_{2}$. In this case the entire budget is invested in the first investment type.

C. Assume next that a firm wants to maximize its profit by finding optimal production level when the price function is uncertain. Assume the uncertain price function is $p(x)=A-B x+\varsigma$, where $A, B>0$ and $\varsigma$ is a white noise with $\operatorname{Var}(\varsigma)=\sigma^{2}$. If the cost function of the firm is $C(x)=a x+b$ with $a, b>0$, then its profit is given as

$$
\pi=x(A-B x+\varsigma)-(a x+b)
$$

with expectation and variance

$$
E(\pi)=x(A-B x)-(a x+b)
$$

and

$$
\operatorname{Var}(\pi)=x^{2} \sigma^{2}
$$

Therefore by assuming constants value of $\alpha$ which is the case with exponential utility function, equation (9) becomes

$$
\pi^{*}=x(A-B x)-(a x+b)+\frac{1-\sqrt{1+4 \alpha^{2} x^{2} \sigma^{2}}}{2 \alpha}
$$

By differentiation,

$$
\frac{\partial \pi^{*}}{\partial x}=A-2 B x-a-\frac{2 \alpha \sigma^{2} x}{\sqrt{1+4 \alpha^{2} x^{2} \sigma^{2}}}=A-2 B x-a-\frac{2 \alpha \sigma^{2}}{\sqrt{\frac{1}{x^{2}}+4 \alpha^{2} \sigma^{2}}}
$$

which strictly decreases in $x$, so $\pi^{*}$ is strictly concave and the first order condition is sufficient for optimality. At $x=0, \frac{\partial \pi^{*}}{\partial x}=A-a$ and as $x \rightarrow \infty, \frac{\partial \pi^{*}}{\partial x}$ converges to $-\infty$ implying that there is a unique optimal solution. If $A \leq a$, then $x_{o p t}=0$, otherwise it is the unique solution of the monotonic equation

$$
A-2 B x-a-\frac{2 \alpha \sigma^{2} x}{\sqrt{1+4 \alpha^{2} x^{2} \sigma^{2}}}=0
$$

which leads to a polynomial equation of degree 4. Standard methods are available for solving the equation (Szidarovszky and Yakowitz, 1978).

\section{Conclusions}

In this paper the certainty equivalent known from the economic literature was reconsidered. A slightly modified proof of the classical formula did provide alternative rules, 
which are more accurate than the classical formula which was illustrated with a computer study. Simple applications illustrated the methodology.

\section{References}

[1] Bellman, R. and L.A.Zadeh (1970) Decision Making in a Fuzzy Environment. Management Science, 17(4), 141-164.

[2] Csábrági A, Molnár M (2011) Role of Non-Conventional Energy Sources in Supplying Future Energy Needs, Bulletin of the Szent István University, Gödöllő (Special Issue: p. 216). (2011)

[3] DeGroot, M.H. (1970) Optimal Stochastic Decisions. New York: McGraw-Hill.

[4] Hatvani I G, Magyar N, Zessner M, Kovács J, Blaschke A P (2014) The Water Framework Directive: Can more information be extracted from groundwater data? A case study of Seewinkel, Burgenland, eastern Austria, Hydrogeology Journal 22:(4) pp. 779-794. (2014)

[5] Kall, P. and S.W. Wallace (1994) Stochastic Programming. Chichester: Wiley.
[6] Kovács J, Kovács S, Magyar N, Tanos P, Hatvani I G, Anda A (2014) Classification into homogeneous groups using combined cluster and discriminant analysis, Environmental Modelling \& Software 57: pp. 52-59. (2014)

[7] Molnár Márk (2014) Opportunities for Hungary under the Stability Reserve of the EU ETS, Journal of Central European Green Innovation 2:(2) pp. 105-114. (2014)

[8] Molnar, S., F. Szidarovszky. Game Theory, Multiobjective Optimization, Conflict Resolution, Differential Games (in Hungarian). Computerbooks, Budapest, Hungary, 2011.

[9] Prekopa, A. (1995) Stochastic Programming. Dordrecht: Kluwer Academic Publishers.

[10] Sargent, T.J. (1979) Macroeconomic Theory. New York: Academic Press.

[11] Szidarovszky, F., M. Gershon and L. Duckstein (1986) Techniques for Multi-objective Decision Making in Systems Management. Amsterdam: Elsevier.

[12] Szidarovszky, F. and S. Yakowitz (1978) Principles and Procedures of Numerical Analysis. New York: Plenum Press. 